\title{
Analytical methods used in estimating the prevalence of HIV/AIDS from demographic and cross-sectional surveys with missing data: a systematic review
}

Neema R. Mosha ${ }^{1,2,3^{*}}$, Omololu S. Aluko' ${ }^{1}$ Jim Todd ${ }^{3,4}$, Rhoderick Machekano ${ }^{1}$ and Taryn Young ${ }^{1}$

\begin{abstract}
Background: Sero- prevalence studies often have a problem of missing data. Few studies report the proportion of missing data and even fewer describe the methods used to adjust the results for missing data. The objective of this review was to determine the analytical methods used for analysis in HIV surveys with missing data.

Methods: We searched for population, demographic and cross-sectional surveys of HIV published from January 2000 to April 2018 in Pub Med/Medline, Web of Science core collection, Latin American and Caribbean Sciences Literature, Africa-Wide Information and Scopus, and by reviewing references of included articles. All potential abstracts were imported into Covidence and abstracts screened by two independent reviewers using pre-specified criteria. Disagreements were resolved through discussion. A piloted data extraction tool was used to extract data and assess the risk of bias of the eligible studies. Data were analysed through a quantitative approach; variables were presented and summarised using figures and tables.

Results: A total of 3426 citations where identified, 194 duplicates removed, 3232 screened and 69 full articles were obtained. Twenty-four studies were included. The response rate for an HIV test of the included studies ranged from 32 to 96\% with the major reason for the missing data being refusal to consent for an HIV test. Complete case analysis was the primary method of analysis used, multiple imputations 11(46\%) was the most advanced method used, followed by the Heckman's selection model 9(38\%). Single Imputation and Instrumental variables method were used in only two studies each, with 13(54\%) other different methods used in several studies. Forty-two percent of the studies applied more than two methods in the analysis, with a maximum of 4 methods per study. Only 6(25\%) studies conducted a sensitivity analysis, while 11(46\%) studies had a significant change of estimates after adjusting for missing data.

Conclusion: Missing data in survey studies is still a problem in disease estimation. Our review outlined a number of methods that can be used to adjust for missing data on HIV studies; however, more information and awareness are needed to allow informed choices on which method to be applied for the estimates to be more reliable and representative.
\end{abstract}

Keywords: Missing data, Non-response, Surveys, HIV/AIDS

\footnotetext{
* Correspondence: nmosha90@yahoo.com

1 Division of Epidemiology and Biostatistics, Faculty of Medicine and Health

Sciences, Stellenbosch University, P.O. Box 241, Francie van Zijl Drive, 7505

Tygerberg, Cape Town, South Africa

${ }^{2}$ Mwanza Intervention Trials Unit, P.O. Box 11936, Isamilo road, Mwanza,

Tanzania

Full list of author information is available at the end of the article
}

C C The Author(s). 2020 Open Access This article is licensed under a Creative Commons Attribution 4.0 International License, which permits use, sharing, adaptation, distribution and reproduction in any medium or format, as long as you give appropriate credit to the original author(s) and the source, provide a link to the Creative Commons licence, and indicate if changes were made. The images or other third party material in this article are included in the article's Creative Commons licence, unless indicated otherwise in a credit line to the material. If material is not included in the article's Creative Commons licence and your intended use is not permitted by statutory regulation or exceeds the permitted use, you will need to obtain permission directly from the copyright holder. To view a copy of this licence, visit http://creativecommons.org/licenses/by/4.0/ The Creative Commons Public Domain Dedication waiver (http://creativecommons.org/publicdomain/zero/1.0/) applies to the data made available in this article, unless otherwise stated in a credit line to the data. 


\section{Background}

Worldwide, the HIV/AIDS epidemic is still a problem. It is estimated that currently, 37 million people are living with HIV (PLHIV), with 70\% of these in sub-Saharan Africa [1]. The estimated HIV prevalence is usually obtained from nationally representative, population studies such as demographic health surveys (DHS). However, surveys often have a problem of missing data, which can be a source of bias and can reduce study precision [2].

Accurate HIV prevalence estimates are important for monitoring and evaluating the ongoing programs, for the prevention and treatment of HIV and the allocation of resources within countries [3]. The available literature and guidelines on reporting observational studies(STROBE) suggest that for results to be efficient, the amount of data missing and methods used for handling the problem must be reported [4,5]. The STROBE guidelines go further and explain the importance of reporting the reasons for missingness, which may include unit non-response, where a study participant or household are missing from the entire study, or item non-response, where some questions are not responded to, or wrongly entered in the database. The common reason for missing data in HIV studies includes the refusal to test or non-response to the survey $[3,6]$. However, few studies report the proportion of missing data or even fewer describes the methods used to adjust for missing data [7].

Most of the published articles for estimating the prevalence and incidence of any diseases are based only on the use of complete case data analysis or available case analysis [8]. A few of the articles describe ad hoc methods such as the use of dummy variable and mean imputation for the estimation of disease prevalence and incidence. And even fewer articles describe more advanced methods for adjusting for missing data, such as inverse probability weighting, instrumental variables and multiple imputations [7, 9].

Many demographic and cross-sectional surveys have been conducted to estimate HIV prevalence and have been reported in peer-reviewed journals, but few recognise the bias that could be present from missing data. Editors and authors need to consider how these estimates have been obtained and how missing data have been addressed. It is important that advanced methods to adjust for missing data are incorporated in the analysis of HIV survey data to reduce the bias in the estimates. Failure to adjust for missing data may result in biased estimates of parameters of interest and can have a negative impact on controlling the epidemic [9]..

This study aimed to conduct a review of articles from HIV surveys with missing data to determine what analytical methods or techniques have been used during, estimating HIV prevalence. Also, to identify the methods used for sensitivity analysis to assess the robustness of the assumptions used.

\section{Methods}

Two guidelines were used during the conducting and reporting this review, the Preferred Reporting Items for Systematic Reviews and Meta-Analyses (PRISMA) [10] and Strengthening the Reporting of Observational Studies in Epidemiology (STROBE) [5].

\section{Eligibility criteria and search strategy}

An information specialist searched five different databases on 13th August 2018. The database list included Medline via PubMed, Web of Science Core Collection, Latin American and Caribbean Sciences Literature, Africa-Wide Information and Scopus. (Additional file 1).

Studies published from population surveys, either demographic or cross-sectional studies from January 2000 to August 2018 on estimating the prevalence of HIV/AIDS written in English were eligible to be included in the review. All articles had to include a statement or paragraph on how missing data or nonresponse was handled during analysis in the abstract.

\section{Study selection procedure}

All potential studies were imported into Covidence screened for their titles and abstracts to identify the relevant studies (Covidence systematic review software, Veritas Health Innovation, Melbourne, Australia. Available at www.covidence.org). Two independent reviewers applied the pre-specified criteria to select abstracts and to reject abstracts that are not relevant, with a third reviewer acting as a tiebreaker. Full text of all selected abstracts were obtained and assessed against the eligibility criteria. Disagreements were resolved through discussion between the two reviewers and the third reviewer.

\section{Data extraction and risk of bias assessment}

Before data extraction, all studies were assessed for the possibility of bias using a tool adapted from Hoy et al. 2012 [7, 11]. The Hoy tool has been designed to assess the risk of bias in population-based prevalence studies; it comprises of 10 domains which allow us to identify the study included if it has a low or high risk of bias. The items include a question that assessed the internal validity on the representativeness of the national or target population, sampling strategy used, the likelihood of non-response and question that assessed the external validity on how data were collected and analysed, reliability and validity of the estimates(Additional file 2). We used Kappa statistics to assess the agreement between the two reviewers on the full text studies included. The values where set as ranges of 0 to 0.20 as slight agreement; 0.21 to 0.40 , fair agreement; 0.41 to 0.60 , 
moderate agreement; 0.61 to 0.80 , substantial agreement; and greater than 0.80 almost perfect agreement.

A piloted data extraction form with structured questions was used to collect data from the included studies independently by the two reviewers. We collected data on year of publication, place of study, type of study, sample size and if adjusted for missing data, how the outcome of interest was analysed, primary analysis and methods used to adjust for missing values. Discrepancies were discussed and resolved; an external reviewer was invited in if the consensus was not achieved from the two reviewers. The data extraction tool used is included as Additional file 3.

\section{Data analysis}

The extracted data were analysed through a quantitative approach. All the variables collected were described and summarised using flow chart and tables. The characteristics of individual studies included were described. Proportions of studies that reported missing values and the methods used to adjust for missing data or selection bias were summarised in the following way. Methods used for analysis were also described and, any other studies that performed sensitivity analyses for any of the methods were also quantified.

\section{Results}

A total of 3426 citations were identified, 194 duplicates removed, 3232 screened, and 69 full articles obtained. The excluded abstracts were not surveys, or were not estimating HIV prevalence, or did not include any missing data methods to estimate HIV. Following full-text eligibility assessment, 24 studies were included while 45 studies were excluded due to not being a survey [12], not measuring HIV prevalence [13], being a methodological study [8], having no missing data methods used during analysis [3], duplicates [3] and 1 study where we could not assess the risk of bias, as it did not show the adjusted HIV prevalence after using the advanced methods for missing data. Table 1 shows the details of the excluded studies and a flow chart of the systematic review is provided in Fig. 1.

\section{Description of included studies}

Out of the24 studies, 12 (50\%) were Demographic Health Survey (DHS) studies [48-60], Seven (29\%) Cross-sectional surveys [52,61-66], three (13\%) population surveys [67-69] and 2(8\%) a mixture of Demographic Health Survey and Aids Indicator surveys [50, 70]. These studies were published between 2006 to 2018, and more than $95 \%$ of the studies were done in subSaharan Africa. The age of the participants ranged from 12 to 64 years, with more women than men participants. Table 2 provides a summary of 10 of the included studies which used a single, unique source of data, and did not use DHS data.

Fourteen studies had multiple sources of data that were analysed. Whereby in other studies datasets were used more than once. All these studies used DHS data from different countries in Sub-Saharan Africa. The most common data set used was from Zambia DHS (2007) and Zimbabwe DHS (2006). A study by Marino et al. used more datasets than any other study (28/32) followed by Hogan et al. (27/32) and Mirsha et al. (14/ 32). Table 3 shows the intersection of data usage from the 14 studies with multiple sources of datasets, including DHS data.

\section{Risk of Bias assessment}

The overall Cohen's kappa coefficient statistic for the two authors screening all the included studies was estimated to be 0.93 . We had a higher risk of bias on domains that assessed the internal validity of the studies compared to domains assessing external validity. Almost all studies had a higher risk of bias on Domain 4 which looked on likelihood of non-response (23/24), followed by Domain 1 which looked on the target population is a close representation of the national population (10/24) (Appendix 4). Only one study had a high risk of bias in terms of domains that looked on external validity (domain 8), which asked if the same mode of data collection was used for all subjects. Additional files 2 and 4 shows in detail all the domain assessed, and results of the assessment done.

\section{Characteristics of the missing data}

Only 21 of the 24 studies reported the response rate for an HIV test. It ranged from 32 to $96 \%$. All the studies gave a reason for the missing data reported, major reason being the participant refused to consent to an HIV test and 8 (33\%) studies identified further missing data from unit-nonresponse Six (25\%) studies reported missing data as a separate outcome, while only $9(38 \%)$ had a result table comparing the participants with complete data and the ones with missing data. Table 4 provides a summary of the mentioned characteristics.

\section{Analytical methods used in the analysis}

All the 24 studies included in the analysis used complete case analysis method as their primary method of analysis. Multiple imputations $11(46 \%)$ was the most advanced method used to adjust for missing data followed by the Heckman's selection model 9(38\%). Single Imputation and Instrumental variables method were used in only two studies each, with 13(54\%) other different methods used in several studies. Ten studies (42\%) applied more than two methods in the analysis, with a maximum of 4 methods in two studies. Table 5 
Table 1 Excluded studies and reasons for exclusion

\begin{tabular}{|c|c|c|}
\hline Reference & Reason for exclusion & $\mathrm{n}(\%)$ \\
\hline $\begin{array}{l}\text { Arpino 2014, Barbosa 2002, Blum 2010, Dagne 2015, DiRienzo 2009, Guan 2017, Huang 2012, } \\
\text { Kenward 2001, Nyirenda 2010, Obare 2010, Patrician 2002, Scharfstein 2003, Sun 2018, } \\
\text { Tian } 2007 \text { [12-24]. }\end{array}$ & Not a survey & $14(31.1)$ \\
\hline $\begin{array}{l}\text { Bärnighausen 2012, Grassly 2004, Hlalele 2008, Kranzer 2008, Liu Y 2015, Liu S 2015, } \\
\text { Mistry 2008, Nelwamondo 2007, Pantanowitz 2009a, Pantanowitz 2009b, Rosinska 2013, } \\
\text { Schomaker 2018, Shah 2014, Westreich 2012, Wirth 2010, Wu } 2001 \text { [25-39]. }\end{array}$ & Do not measure HIV prevalence & $16(35.6)$ \\
\hline $\begin{array}{l}\text { Boerma 2003, Brookmeyer 2010, Clark 2012; Garcia-Calleja 2006, Gouws 2008, Hund 2013, } \\
\text { Korenromp 2013, Larmarange } 2014 \text { [2, 6, 40-44]. }\end{array}$ & Methodological article & $8(17.8)$ \\
\hline Alkema 2008, Montana 2008, Kayibanda 2011 [45-47]. & $\begin{array}{l}\text { No missing data methods used in the } \\
\text { analysis }\end{array}$ & $3(6.7)$ \\
\hline McGovern 2015a, Obare 2010, Pentanowitz 2009a [23, 33, 48]. & Duplicate & $3(6.7)$ \\
\hline $\operatorname{Ng} 2013$ [49]. & Could not assess the risk of bias & $1(2.2)$ \\
\hline
\end{tabular}

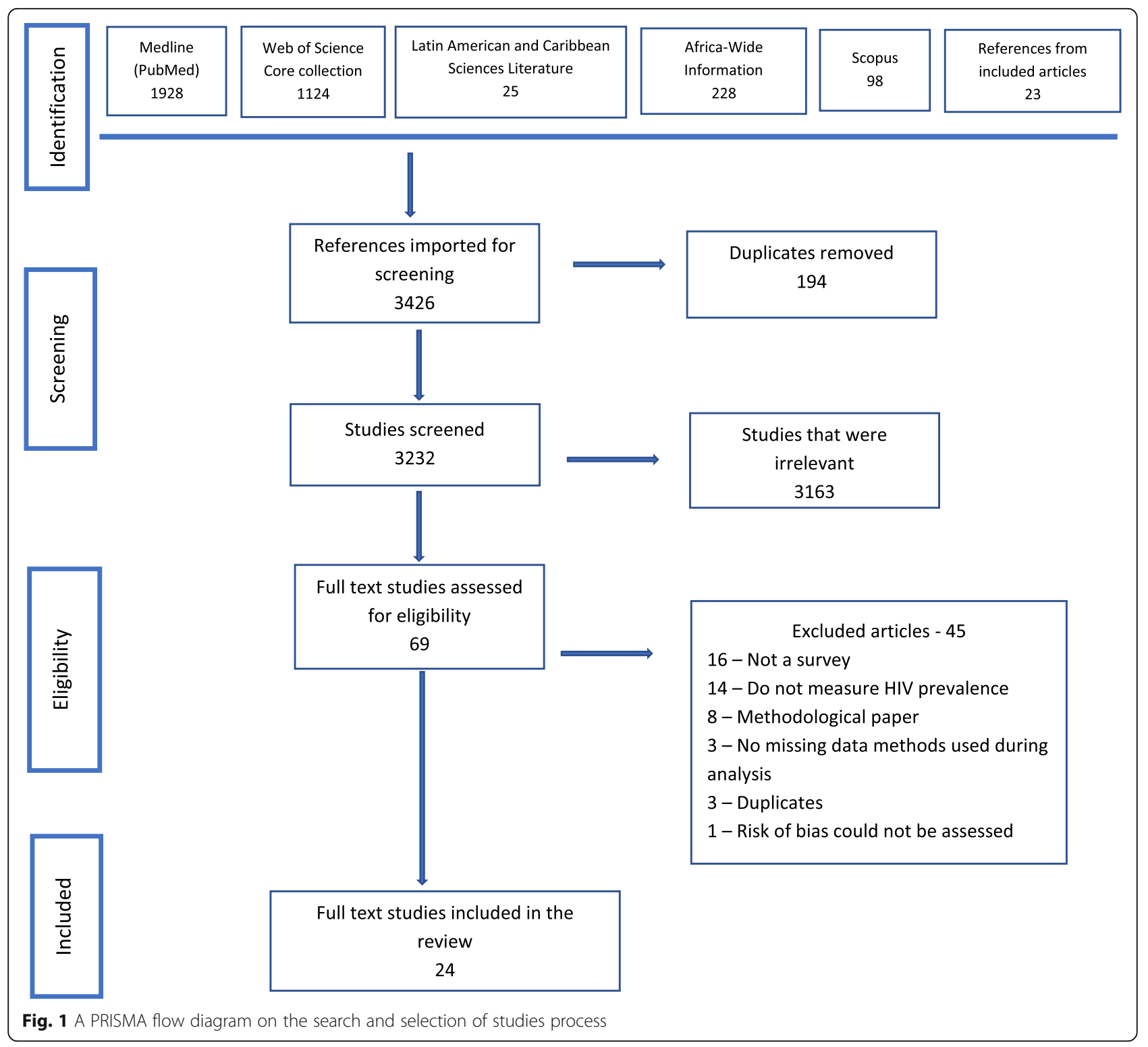


Table 2 Description of included studies which used only one source of data

\begin{tabular}{|c|c|c|c|c|c|c|c|}
\hline$\overline{\text { No }}$ & Study ID & Country & Year of survey & Year of publication & Sample size & Age of included participants & Type of study \\
\hline 1 & Floyd [61] & Malawi & $2006-2010$ & 2013 & 17,000 & $\geq 15$ & Cross-sectional survey \\
\hline 2 & Harling [71] & South Africa & 2012 & 2017 & 42,357 & $\geq 15$ & Population Survey \\
\hline 3 & Jessens [62] & Namibia & 2008-2009 & 2014 & 1992 & $\geq 12$ & Cross-sectional survey \\
\hline 4 & Kendall [63] & Angola & 2011 & 2014 & 792 & $\geq 18$ & Cross-sectional survey \\
\hline 5 & Kerr [65] & Brazil & 2016 & 2018 & 4176 & $\geq 18$ & Cross-sectional survey \\
\hline 6 & Kerr [64] & Brazil & 2009 & 2013 & 3859 & $\geq 18$ & Cross-sectional survey \\
\hline 7 & Leacy [68] & Zambia & 2006-2010 & 2016 & 34,446 & $\geq 18$ & Population survey \\
\hline 8 & McGovern [69] & South Africa & 2009 & 2015 & 25,392 & $\geq 15$ & Population survey \\
\hline 9 & Reiners [52] & Ethiopia & 2003-2004 & 2009 & 1650 & $\geq 16$ & Cross-sectional survey \\
\hline 10 & Ziraba [66] & Kenya & 2006-2007 & 2010 & 4767 & $\geq 15$ & Cross-sectional survey \\
\hline
\end{tabular}

describes the methods used to adjust for missing data on estimating HIV prevalence.

Only 1 study mentioned the pattern identified of the missing data, while more than half $13(53 \%)$ of the studies stated the mechanism assumed in the analysis. Of the 13 studies that mentioned the mechanism used during analysis, all studies assumed data to be MCAR for the complete analysis, 11 assumed data to be MNAR, ten assumed data to be MAR and seven studies assumed both MAR and MNAR. For the studies that used Multiple imputation method, only 3 (27\%) stated the number of imputed data sets in the analysis, but seven (64\%) mentioned the variables used in the imputation model. On assessing the robustness of the results only $6(25 \%)$ studies conducted a sensitivity analysis, while 11(46\%) studies had a significant change of estimates after adjusting for missing data. Table 6 provides details on the different aspects of the analysis strategy and methods.

\section{Discussion}

We identified 69 citations that fulfilled our eligibility criteria on this HIV topic with only 24 studies addressing the missing data problem on the estimation of HIV prevalence during analysis. The same trend of fewer studies addressing the missing data problem is observed in other design like clinical trials and HIV longitudinal studies measuring different outcome [72]. The major reason for the missingness was reported to be a refusal to consent for an HIV test, and with complete case analysis be the primary method of analysis used. Multiple imputations and Heckman's selection models were the major methods used to adjust for missing data, with $46 \%$ of studies showing a significant change of estimates after adjustments. Only a quarter of the included studies conducted a sensitivity analysis to assess the robustness of the results.

There was a good agreement between authors regarding the risk of bias, for all the included studies we had a high risk of bias on the domains assessing the internal validity of the studies compared to domains assessing the external validity, i.e. on the likelihood of nonparticipation. This may be because one criterion for the inclusion to the review was the study should have a line addressing the missing data or non-response problem.

The STROBE guideline [5] recommends that authors to report the amount of missing data, methods of handling missing data and the reasons for missingness $\mathrm{s}$. However, of all included studies, only one was published before the STROBE guidelines in 2007, while others were published afterwards, and we found out that in most of the included studies provided the amount of missing data, with the corresponding reasons for missingness however, very few studies explored the differences between the participants with complete data and with missing data which can be used as the bases of examining the MCAR assumption.

The included studies used different methods for missing data analysis, and these ranged from ad hoc (complete case and single imputation) to advanced methods assuming MAR or MNAR mechanism (e.g., multiple imputations). Multiple imputations were the common method used despite that in most of the studies the methodology behind it was not clearly explained like the algorithm followed during imputation, number of imputed dataset and details on the imputation model. Provision of this information helps the replication of the methods and assessment of the results.

We observe an increase of the HIV prevalence estimates after adjusting for the missing data, demonstrating the presence of downward bias if complete case analysis is used The differences were significant in some studies $[58,71]$, and this suggests there might be underestimating of HIV prevalence if missing data are ignored.

All the applied methods had the shortcoming of its application considering the mechanism followed since there is no proof that missing data were MAR or MNAR. Heckman's selection models and application of instrumental variables where the methods tried to 


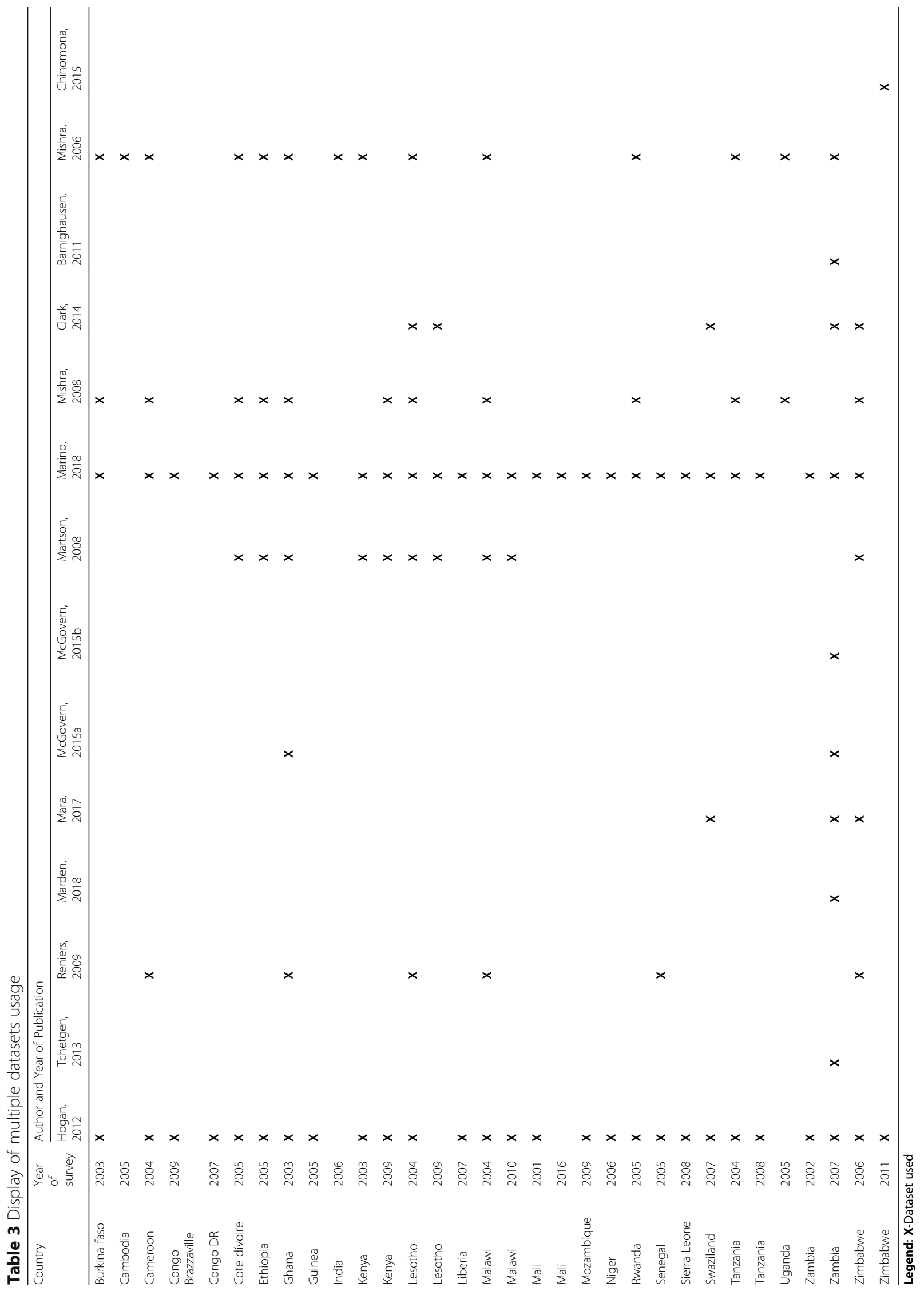


Table 4 Summary of the missing data characteristics $(n=24)$

\begin{tabular}{|c|c|c|}
\hline CHARACTERISTICS & $\mathrm{n}$ & $\%$ \\
\hline \multicolumn{3}{|l|}{ Response rate reported } \\
\hline Yes & 21 & 88 \\
\hline No & 3 & 22 \\
\hline \multicolumn{3}{|l|}{ Response rate reported } \\
\hline$<70 \%$ & 2 & 9 \\
\hline $70-80 \%$ & 10 & 48 \\
\hline$>80 \%$ & 9 & 43 \\
\hline \multicolumn{3}{|c|}{ Reasons for missing data reported } \\
\hline Yes & 24 & 100 \\
\hline No & 0 & 0 \\
\hline \multicolumn{3}{|l|}{ What were the reasons reported } \\
\hline Refusal to test for HIV & 24 & 100 \\
\hline Absence & 3 & 13 \\
\hline Technical problems & 1 & 4.2 \\
\hline \multicolumn{3}{|c|}{ Type of missing data mentioned } \\
\hline Unit non-response & 8 & 33 \\
\hline Unit and Item non-response & 16 & 67 \\
\hline \multicolumn{3}{|c|}{ Missing data reported as a separate outcome } \\
\hline Yes & 6 & 25 \\
\hline No & 18 & 75 \\
\hline \multicolumn{3}{|c|}{$\begin{array}{l}\text { A summary table to compare participants with complete data vs } \\
\text { incomplete data }\end{array}$} \\
\hline Yes & 9 & 38 \\
\hline No & 15 & 62 \\
\hline
\end{tabular}

explore the deviation of MAR to the possibility of MNAR assumption although a lack of suitable selection or instrumental variable impacts their applicability [57, 71]. The use of doubly robust methods and extension of Heckman's selection models are the current methods identified as suitable when data are assumed to be MNAR. With the assumption that the missing data on HIV prevalence studies not being MAR, and the possibility of MNAR $[54,68]$, it is important to explore more methods than identified from this review.

Further to the analysis, a report from National Research Council (NRC) [73] explains the importance of conducting sensitivity analysis to assess the robustness of the results and conclusion of the assumptions used on the application of methods used to adjust for missing data. However, Only a quarter of the included studies performed a sensitivity analysis.... This does not differ with results provided by other reviews on missing dat, that very few studies assessed the robustness of the results regardless of the design $[74,75]$.

This is the first systematic review exploring the methods used in addressing the missing data problem on estimating HIV prevalence, however these results can
Table 5 Missing data methods used in the analysis

\begin{tabular}{lll}
\hline CHARACTERISTICS & $\mathrm{n}$ & $\%$ \\
\hline Major methods used for analysis & 24 & 100 \\
Complete case analysis & 2 & 8 \\
Single imputation & 11 & 46 \\
Multiple Imputation & 2 & 8 \\
Instrumental variables & 9 & 38 \\
Heckman's selection model & 13 & 54 \\
Other methods & & \\
Other methods used & 2 & 8 \\
Age standardization & 1 & 4 \\
Upper bounds and lower bounds & 2 & 8 \\
Copulae models & 1 & 4 \\
Logistic prediction models & 1 & 4 \\
Refusal rate adjustment & 1 & 4 \\
Mobility rate adjustment & 1 & 4 \\
Random effect bias model & 1 & 4 \\
HIV self-report imputation & 1 & 8 \\
Prevalence ratio inflation factor & 1 & 4 \\
HIV risk ratio adjustment & 1 & 4 \\
Network imputation using recruitment chain & 1 & 4 \\
Conditional probability equations & 1 & 4 \\
Maximum number of methods used per study & & \\
2 & 1 & 58 \\
3 & & 4 \\
\hline
\end{tabular}

only be generalizable to studies where the focus is on missing data This review will guide us in future application of these methods on real datasets from a population-based study conducted in North-West Tanzania and estimate the amount of bias caused by the missing data. Also, we will extend the methods assuming data being MNAR with further assessment by using a sensitivity analysis approach.

\section{Conclusion}

This review aimed to look at surveys to determine what analytical methods or technique have been used to address the missing data problem on estimating HIV prevalence. From the studies included we saw that several methods can be used when data are not missing completely at random,. However, studies often report very little information on the steps, theories, assumptions and sensitivity of the reported results. .

All methods used for handling missing data in the included studies produced different estimates from the primary analysis, and in some studies, the difference was large. These differences highlight the need for considering using more advance methods when facing the 
Table 6 Further information on the analysis and results conclusion provided

CHARACTERISTICS $n \quad$ n

Missing data pattern stated in the analysis

Yes

No

$\begin{array}{ll}1 & 4 \\ 23 & 96\end{array}$

Missing data mechanism stated in the analysis

$\begin{array}{lll}\text { Yes } & 13 & 54 \\ \text { No } & 11 & 46\end{array}$

Reported assumption or mechanism used in the analysis $(n=13)$

$\begin{array}{lll}\text { MCAR } & 13 & 100 \\ \text { MAR } & 8 & 62 \\ \text { MNAR } & 9 & 75 \\ \text { MAR and MNAR } & 7 & 58\end{array}$

The simulation method used before the analysis

Yes

No

$\begin{array}{ll}4 & 17 \\ 20 & 83\end{array}$

Details on the multiple Imputation $\operatorname{method}(n=11)$

Number of imputations stated

$3 \quad 27$

Variables included in the imputation model stated

Additional file 2. Data extraction tool.

Additional file 3. Risk of Bias assessment domains.

Additional file 4. Risk of bias assessment table.

Abbreviations

DHS: Demographic Health Surveys; MAR: Missing at Random; MCAR: Missing Completely at Random; MNAR: Missing Not at Random; NRC: National Research Council; STROBE: Strengthening the Reporting of Observational Studies in Epidemiology

Acknowledgements

We acknowledge Anel Schoonees for helping with searching of the studies, and Traci Naidoo for the logistic support.

\section{Authors' contributions}

All authors have read and approved the manuscript for publication. NM: Conceived the research question, developed protocol and search strategy, reviewed articles, extract and analyses the data, interpret results and manuscript development. OA: Review articles, extract and analyses the data, interpret results and manuscript development. JT: Co-developed the protocol, approved the search strategy, aided with any extracted data ambiguities and contributed to revisions of drafts of the manuscript as well as providing the final approval of the version to be published. RM: Contributed to proto$\mathrm{col}$ and revisions of drafts of the manuscript as well as providing the final approval of the version to be published. TY: Helped in conceiving the research questions, co-developed protocol, approved search strategy, interpreted results, manuscript development, and provided the final approval of the version to be published.

If the selection model used what was the selection variables? $(n=$ 9)

Interviewer identity 9100

Household visited on the first day of fieldwork

If instrumental variable used what was the variables? $(n=2)$

Interviewer identity

2

Sensitivity analysis performed

Yes

No

$6 \quad 25$

$18 \quad 75$

Changes reported to conclusion

No

Non-significant changes

Significant changes

28

1146

1146

problem of missing data in surveys and population studies to avoid producing biased results.

A further extension of this work is needed to compare the effectiveness of the estimates, and the amount of bias remaining from the available methods for dealing with missing data. Awareness is an important aspect of ensuring that these methods are applied appropriately, and the right choices are made considering the reasons, patterns and mechanism of the missing data..

\section{Supplementary information}

Supplementary information accompanies this paper at https://doi.org/10. 1186/s12874-020-00944-w.

Additional file 1. Search Strategy.

\section{Funding}

This study was supported through the DELTAS Africa Initiative SSACAB (Grant No. 107754/Z/15/Z). The DELTAS Africa Initiative is an independent funding scheme of the African Academy of Sciences (AAS) Alliance for Accelerating Excellence in Science in Africa (AESA) and is supported by the New Partnership for Africa's Development Planning and Coordinating Agency (NEPAD Agency) with funding from the Wellcome Trust (Grant No. 107754/Z/15/Z) and the UK government. The views expressed in this publication are those of the authors and not necessarily those of the AAS, NEPAD Agency, Wellcome Trust or the UK government.

Availability of data and materials

Dataset used in the analysis will be made available from the corresponding author on reasonable request.

Ethics approval and consent to participate

Not applicable.

\section{Consent for publication}

No consent was required.

\section{Competing interests}

The authors declare that they have no competing interests.

\section{Author details}

${ }^{1}$ Division of Epidemiology and Biostatistics, Faculty of Medicine and Health Sciences, Stellenbosch University, P.O. Box 241, Francie van Zijl Drive, 7505 Tygerberg, Cape Town, South Africa. ${ }^{2}$ Mwanza Intervention Trials Unit, P.O. Box 11936, Isamilo road, Mwanza, Tanzania. ${ }^{3}$ National Institute for Medical Research, Mwanza Centre, P.O. Box 1462, Isamilo road, Mwanza, Tanzania. ${ }^{4}$ London School of Hygiene and Tropical Medicine, Keppel St, Bloomsbury, London WC1E 7HT, UK.

Received: 8 October 2019 Accepted: 28 February 2020

Published online: 14 March 2020

References

1. UNAIDS. UNIADS Report [Internet]. Programme on HIV/AIDS. 2017. Available from: http://www.unaids.org/sites/default/files/media_asset/20170720_Data_ book_2017_en.pdf. 
2. Boerma IT, Ghys PD, Walker N. Estimates of HIV-1 prevalence from national population-based surveys as a new gold standard. Lancet. 2003;362:1929-31.

3. Gouws E, Mishra V, Fowler TB. Comparison of adult HIV prevalence from national population-based surveys and antenatal clinic surveillance in countries with generalised epidemics : implications for calibrating surveillance data. Sex Transm Infect. 2008;84(Suppl I):17-23.

4. Shamliyan T, Kane RL, Dickinson S. A systematic review of tools used to assess the quality of observational studies that examine incidence or prevalence and risk factors for diseases. J Clin Epidemiol. 2010;63(10): 1061-70.

5. von Elm E, Altman DG, Egger M, Pocock SJ, Gøtzsche PC, Vandenbroucke $J P$. The strengthening the reporting of observational studies in epidemiology (STROBE) statement: guidelines for reporting observational studies. Int J Surg. 2014;12(12):1495-9.

6. Garcia-Calleja JM, Gouws E, Ghys PD. National population based HIV prevalence surveys in sub-Saharan Africa : results and implications for HIV and. Sex Transm Infect. 2006:64-70.

7. Eekhout I, De Boer MR, Twisk JWR, De Vet HCW, Heymans MW. Missing data: a systematic review of how they are reported and handled. Epidemiology. 2012;23(5):729-32.

8. Pigott TD. A review of methods for missing data. Educ Res Eval. 2001;7(4): 353-83.

9. Schafer JL, Graham JW. Missing data: Our view of the state of the art. Psychol Methods. 2002;7(2):147-177. 47.

10. Stroup DF, Berlin JA, Morton SC, Olkin I, Williamson GD, Rennie D, et al. Meta-analysis of observational studies. Mod Methods Epidemiol. 2008; 283(15):173-89.

11. Hoy D, Brooks P, Woolf A, Blyth F, March L, Bain C, et al. Assessing risk of bias in prevalence studies : modification of an existing tool and evidence of interrater agreement. J Clin Epidemiol. 2012;65(9):934-9.

12. Scharfstein DO, Irizarry RA. Generalized additive selection models for the analysis of studies with potentially nonignorable missing outcome data. Biometrics. 2003:59(3):601-13.

13. Tian $\mathrm{GL}$, Tan M, Ng KW. An exact non-iterative sampling procedure for discrete missing data problems. Stat Neerl. 2007;61 (2):232-42.

14. Arpino B, De Cao E, Peracchi F. Using panel data for partial identification of human immunodeficiency virus prevalence when infection status is missing not at random. J R Stat Soc. 2014;177(3):587-606.

15. Barbosa MTS, Struchiner CJ. The estimated magnitude of AIDS in Brazil: a delay correction applied to cases with lost dates. Cad saúde pública / Ministério da Saúde, Fundação Oswaldo Cruz, Esc Nac Saúde Pública. 2002; 18(1):279-85.

16. Sun BL, Tchetgen Tchetgen EJ. On inverse probability weighting for nonmonotone missing at random data. J Am Stat Assoc. 2018;113(521): 369-79.

17. Blum MGB, Tran VC. HIV with contact tracing: a case study in approximate Bayesian computation. Biostatistics. 2010;11(4):644-60.

18. Dagne GA, Huang Y. Bayesian two-part tobit models with leftcensoring, skewness, and nonignorable missingness. J Biopharm Stat. 2015;25(4):714-30

19. Dirienzo AG. Flexible regression model selection for survival probabilities: with application to AIDS. Biometrics. 2009;65(4):1194-202.

20. Guan Z, Qin J. Empirical likelihood method for non-ignorable missing data problems. Lifetime Data Anal. 2017;23(1):113-35.

21. Kenward MG, Molenberghs $G$, Verbeke $G$. Sensitivity analysis for incomplete data. Stat Modelling. 2001;2802(1):31-48.

22. Nyirenda M, Zaba B, Bärnighausen T, Hosegood V, Newell ML. Adjusting HIV prevalence for survey non-response using mortality rates: An application of the method using surveillance data from rural south africa. PLoS One. 2010; $5(8)$.

23. Obare F. Nonresponse in repeat population based voluntary counselling and testing for HIV in rural Malawi. Demography. 2010;47(3):651-65.

24. Patrician PA. Multiple imputation for missing data. Res Nurs Health. 2002; 25(1):76-84

25. Bärnighausen T, Tanser F, Malaza A, Herbst K, Newell ML. HIV status and participation in HIV surveillance in the era of antiretroviral treatment: a study of linked population-based and clinical data in rural South Africa. Trop Med Int Heal. 2012;17(8):103-10.

26. Grassly NC, Morgan M, Walker N, Garnett G, Stanecki KA, Stover J, et al. Uncertainty in estimates of HIV/AIDS: the estimation and application of plausibility bounds. Sex Transm Infect. 2004;80(SUPPL. 1):31-8.
27. Halele N, Nelwamondo F, Marwala T. Imputation of missing data using PCA. Neuro-Fuzzy ICONIP. 2008:485-92.

28. Kranzer K, McGrath N, Saul J, Crampin AC, Jahn A, Malema S, et al. Individual, household and community factors associated with HIV test refusal in rural Malawi. Trop Med Int Heal. 2008;13(11):1341-50.

29. Liu Y, Anindya D. Multiple imputation by fully conditional specification for dealing with missing data in a large epidemiologic study. Int J Stat Med Res. 2015;4(3):287-95.

30. Liu SH, Erion G, Novitsky V, De Gruttola V. Viral genetic linkage analysis in the presence of missing data. PLoS One. 2015;10(8):1-14.

31. Mistry J, Nelwamondo F, Marwala T. Estimating missing data and determining the confidence of the estimate data. Proc - 7th Int Conf Mach Learn Appl ICMLA 2008. 2008:752-5.

32. Nelwamondo FV, Mohamed S, Marwala T. Missing data: a comparison of neural network and expectation maximization techniques. Curr Sci. 2007; 93(11):1514-21.

33. Pantanowitz A, Marwala T. Missing data imputation through the use of the random forest algorithm. Adv Intell Soft Comput. 2009;61(AISC):53-62.

34. Rosinska M, Janiec J, Niedźwiedzka-Stadnik M. Increase of new HIV diagnoses among men who have sex with men in Poland, 2000 to 2011. Eurosurveillance. 2013:18(48):1-9.

35. Schomaker M, Heumann C. Bootstrap inference when using multiple imputation. Stat Med. 2018;37(14):2252-66.

36. Shah AD, Bartlett JW, Carpenter J, Nicholas O, Hemingway H. Comparison of random forest and parametric imputation models for imputing missing data using MICE: a CALIBER study. Am J Epidemiol. 2014;179(6):764-74.

37. Westreich D. Berkson's bias, selection bias, and missing data. Bone. 2008; 23(1):1-7.

38. Wirth KE, Tchetgen Tchetgen EJ, Murray M. Adjustment for Missing Data in complex surveys using Doubly Robust Estimation. Epidemiology. 21(6):86371.

39. Wu H, Wu L. A multiple imputation method for missing covariates in nonlinear mixed-effects models with application to HIV dynamics. Stat Med. 2001;20(12):1755-69.

40. Brookmeyer R. Measuring the HIV/AIDS epidemic: approaches and challenges. Epidemiol Rev. 2010;32(1):26-37.

41. Clark SJ, Houle B. Evaluation of Heckman selection model method for correcting estimates of HIV prevalence from sample surveys. Soc Sci. 2012;120

42. Hund L, Pagano M. Estimating HIV prevalence from surveys with low individual consent rates: annealing individual and pooled samples. Emerg Themes Epidemiol. 2013;10(1):1-10.

43. Korenromp EL, Gouws E, Barrere B. HIV prevalence measurement in household surveys: is awareness of HIV status complicating the gold standard? Aids. 2013;27(2):285-7.

44. Larmarange J, Bendaud V. HIV estimates at second subnational level from national population-based surveys. Aids. 2014;28(September):S469-76.

45. Alkema L, Raftery AE, Brown T. Bayesian melding for estimating uncertainty in national HIV prevalence estimates. Sex Transm Infect. 2008;84(SUPPL. 1):11-6.

46. Montana LS, Mishra V, Hong R. Comparison of HIV prevalence estimates from antenatal care surveillance and population-based surveys in subSaharan Africa. Sex Transm Infect. 2008:84(SUPPL. 1):78-84.

47. Kayibanda JF, Alary M, Bitera R, Mutagoma M, Kabeja A, Hinda R, et al. Use of routine data collected by the prevention of mother-to-child transmission program for HIV surveillance among pregnant women in Rwanda: opportunities and limitations. AIDS Care - Psychol Socio-Medical Asp AIDS/ HIV. 2011;23(12):1570-7.

48. McGovern ME, Bärnighausen T, Salomon JA, Canning D. Using interviewer random effects to remove selection bias from HIV prevalence estimates data analysis, statistics and modelling. BMC Med Res Methodol. 2015;15(1): $1-11$.

49. Ng M, Gakidou E, Murray CJL, Lim SS. A comparison of missing data procedures for addressing selection bias in HIV sentinel surveillance data. Popul Health Metrics 2013;11(1):1-10.

50. Mishra V, Vaessen M, Boerma JT, Arnold F, Way A, Barrere B, et al. HIV testing in national population-based surveys: experience from the demographic and health surveys. Bull World Health Organ. 2006;84(7):537-45.

51. Tchetgen Tchetgen EJ, Wirth KE. A general instrumental variable framework for regression analysis with outcome missing not at random. Biometrics. 2013;73(4):1123-31. 
52. Reniers G, Araya T, Berhane Y, Davey G, Sanders EJ. Implications of the HIV testing protocol for refusal bias in seroprevalence surveys. BMC Public Health. 2009;9:1-9.

53. Marden JR, Wang L, Tchetgen EJT, Walter S, Glymour MM, Wirth KE. Implementation of instrumental variable bounds for data missing not at random. Epidemiology. 2018;29(3):364-8.

54. Marra G, Radice R, Bärnighausen T, Wood SN, McGovern ME. A simultaneous equation approach to estimating HIV prevalence with nonignorable missing responses. J Am Stat Assoc. 2017;112(518):484-96.

55. Mcgovern ME, Marra G, Radice R, Studies D. Copula approach applied to estimating HIV prevalence. Epedemiology. 2016;26(2):229-37.

56. Marino M, Pagano M. Role of survey response rates on valid inference: an application to HIV prevalence estimates. Emerg Themes Epidemiol. 2018; 15(1):1-11.

57. Clark SJ, Houle B. Validation, replication, and sensitivity testing of Heckmantype selection models to adjust estimates of HIV prevalence. PLoS One. 2014;9(11).

58. Bärnighausen T, Bor J, Wandira-Kazibwe S, Canning D. Correcting HIV prevalence estimates for survey nonparticipation using heckman-type selection models. Epidemiology. 2011;22(1):27-35.

59. Chinomona A, Mwambi H. Multiple imputation for non-response when estimating HIV prevalence using survey data biostatistics and methods. BMC Public Health. 2015;15(1):1-10.

60. Mishra V, Barrere B, Hong R, Khan S. Evaluation of bias in HIV seroprevalence estimates from national household surveys. Sex Transm Infect. 2008; 84(SUPPL. 1):63-70.

61. Floyd S, Molesworth A, Dube A, Crampin AC, Houben R, Chihana M, et al. Underestimation of HIV prevalence in surveys when some people already know their status, and ways to reduce the bias. AIDS. 2013:27:233-42.

62. Jassens W, van der Gaag J, Rinke de Wit TF, Tanovic Z. Refusal Bias in the Estimation of HIV Prevalence : Demography. 2014;51(3):1131-57.

63. Kendall C, Kerr LRFS, Mota RMS, Cavalcante S, Macena RHM, Chen S, et al. Population size, HIV, and behavior among MSM in Luanda, Angola: challenges and findings in the first ever HIV and syphilis biological and behavioral survey. J Acquir Immune Defic Syndr. 2014;66(5):544-51.

64. Kerr LRFS, Mota RS, Kendall C, Pinho ADA, Mello MB, Guimarães MDC, et al. HIV among MSM in a large middle-income country. Aids. 2013;27(3):427-35.

65. Kerr L, Kendall C, Guimarães MDC, Mota RS, Veras MA, Dourado I, et al. HIV prevalence among men who have sex with men in Brazil: results of the 2nd national survey using respondent-driven sampling. Med (United States). 2018;97(1S):S9-15.

66. Ziraba AK, Madise NJ, Matilu M, Zulu E, Kebaso J, Khamadi S, et al. The effect of participant nonresponse on HIV prevalence estimates in a population-based survey in two informal settlements in Nairobi city. Popul Health Metrics. 2010;8:1-10.

67. Harling G, Moyo S, Chb MB, Mcgovern ME, Mabaso M, Marra G, et al. National South African HIV prevalence estimates robust despite substantial test non-participation. South African Med J. 2017;107(7):590-4.

68. Leacy FP, Floyd S, Yates TA, White IR. Analyses of sensitivity to the missingat-random assumption using multiple imputation with delta adjustment: application to a tuberculosis/HIV prevalence survey with incomplete HIVstatus data. Am J Epidemiol. 2017;185(4):304-15.

69. McGovern ME, Marra G, Radice R, Canning D, Newell ML, Bärnighausen T. Adjusting HIV prevalence estimates for non-participation: $\mathrm{N}$ application to demographic surveillance. J Int AIDS Soc. 2015;18(1):1-11.

70. Marston M, Harriss K, Slaymaker E. Non-response bias in estimates of HIV prevalence due to the mobility of absentees in national population-based surveys: a study of nine national surveys. Sex Transm Infect. 2008;84(SUPPL. 1):71-7.

71. Harling G, Moyo S, McGovern ME, Mabaso M, Marra G, Bärnighausen T, et al. National South African HIV prevalence estimates robust despite substantial test non-participation. South African Med J. 2017;107(7):590-4.

72. Akl EA, Kahale LA, Agoritsas T, Brignardello-Petersen R, Busse JW, CarrascoLabra A, et al. Handling trial participants with missing outcome data when conducting a meta-analysis: a systematic survey of proposed approaches. Syst Rev. 2015;4(1):98.

73. National Research Council of the National Academies. The Prevention and Treatment of Missing Data in Clinical Trials Panel on Handling Missing Data in Clinical Trials ; National Research 2010. 83-106 p.

74. Harel O, Pellowski J, Kalichman S. Are we missing the importance of missing values in HIV prevention randomized clinical trials? Rev Recomm AIDS Behav. 2012;16(6):1382-93.
75. Akl EA, Carrasco-labra A, Brignardello-petersen R, Neumann I, Johnston BC Sun $X$, et al. Reporting, handling and assessing the risk of bias associated with missing participant data in systematic reviews : a methodological survey. BMJ Open. 2015;5:1-8.

\section{Publisher's Note}

Springer Nature remains neutral with regard to jurisdictional claims in published maps and institutional affiliations.

\section{Ready to submit your research? Choose BMC and benefit from:}

- fast, convenient online submission

- thorough peer review by experienced researchers in your field

- rapid publication on acceptance

- support for research data, including large and complex data types

- gold Open Access which fosters wider collaboration and increased citations

- maximum visibility for your research: over $100 \mathrm{M}$ website views per year

At BMC, research is always in progress.

Learn more biomedcentral.com/submissions 